\title{
Magneto-Optic Hybrid 3-D Sensor for Surgical Navigation
}

\author{
Masahiko Nakamoto ${ }^{1,2}$, Yoshinobu Sato ${ }^{2,1}$, Yasuhiro Tamaki ${ }^{3}$, \\ Hiroaki Nagano ${ }^{3}$, Masaki Miyamoto ${ }^{1,2}$, Toshihiko Sasama ${ }^{1,2}$, Morito Monden ${ }^{3}$, \\ and Shinichi Tamura ${ }^{2,1}$ \\ 1 Department of Informatics and Mathematical Science \\ Graduate School of Engineering Science, Osaka University \\ nakamoto@image.med.osaka-u.ac.jp, yoshi@image.med.osaka-u.ac.jp \\ 2 Division of Functional Diagnostic Imaging \\ 3 Department of Surgery and Clinical Oncology \\ Osaka University Graduate School of Medicine
}

\begin{abstract}
The objective of the work described in this paper was to develop an accurate three dimensional (3-D) sensory system without a lineof-sight requirement for surgical navigation inside the body. Although magnetic sensors seem to be particularly suitable for this purpose, their accuracy is affected by metallic objects, which can hardly be avoided in a surgical environment. We propose a new magneto-optic hybrid 3-D sensor configuration that overcomes this limitation. Unlike previous hybrid systems, both the receiver and transmitter of the magnetic sensor are mobile, thereby permitting them to be positioned flexibly and adaptively so as to minimize inaccuracies arising from the presence of peripheral metallic objects. The 3-D position and orientation of the transmitter are measured by an optical sensor in order to accurately track the transformation between the coordinate systems of the magnetic and optical sensors. The effects of the distance between the receiver and the transmitter and their respective distances from metallic objects on the accuracy of the system were evaluated by experiments both in the laboratory and in the operating room.
\end{abstract}

\section{Introduction}

Although optical three-dimensional (3-D) position sensors are widely used in surgical navigation systems on account of their high degree of accuracy and acceptable speed [1]-[3], their line-of-sight requirement means that they have inherent limitations in obtaining 3-D information relating to flexible instruments and imaging devices inside the body. Magnetic 3-D position sensors [3] seem to be particularly suitable for acquiring such 3-D positions, and miniature magnetic sensors have recently been developed (for example, Biosense, Johnson \& Johnson) that enable the 3-D position and orientation to be obtained even at the tip of a catheter or a flexible endoscope. However, magnetic sensors have the drawback that their accuracy is affected by metallic objects, which are inevitably in a surgical environment. Our aim is to overcome the limitations of optical and magnetic sensors by combining them in a hybrid system. 
In the operating room (OR), the OR table and surgical instruments are considered to be the major causes of inaccuracies in magnetic systems. Since the OR table has a large surface area, the magnetic sensor performance is considerably affected at all time. Although surgical instruments are much smaller, they can give rise to inaccuracies when placed in the vicinity of the measurement area. Unlike the case of the OR table, distortion caused by surgical instruments varies with their arrangement during the operation. In previous work on optical and magnetic sensor hybridization [4]-[6], magnetic sensor distortion caused by metallic objects in measured 3-D positions has been corrected by using pre-calibrated data. However, since such distortion depends on the specific environment, time-consuming calibration is needed for each environment, and it is particularly difficult to calibrate for surgical instrument distortion before an operation. In addition, distortion correction is not usually carried out in 3-D orientations because of its difficulty.

In the work reported here, we designed and evaluated a new magneto-optic hybrid sensor configuration. Unlike previous configurations, which employ a fixed transmitter for the magnetic system, we arrange for the transmitter to be flexibly positioned so that the accuracy is sufficient without the need for any correction by pre-calibration. The $3-\mathrm{D}$ position and orientation of the transmitter are measured by an optical sensor to accurately track the transformation between the coordinate systems of the magnetic and optical sensors. The accuracy of a magnetic sensor typically depends on the ratio of the distance between the receiver and transmitter to the distance between them and a metallic object. Hence, inaccuracies arising from the presence of metallic objects can be expected to be sufficiently reduced by adaptively arranging the transmitter so that the former distance is small while the latter is large. This strategy can also be expected to reduce the effects of surgical instruments placed in the vicinity of the measurement area.

Our goal is to provide surgeons with augmented reality visualization by utilizing 3-D ultrasound images [1]. Since the probe is usually scanned from the incision opening to the internal region, the line-of-sight requirement is a significant limitation. This is the main reason for using a magneto-optic hybrid sensor. On the other hand, an optical sensor is suitable for obtaining the position and orientation of the camera that takes video images of a patient for augmented reality. Like previous hybrid systems, our hybrid configuration allows complementary use of both types of sensors, but they are combined in a way that reduces the inaccuracy of the magnetic sensor without the need for time-consuming precalibration. We evaluated the accuracy of the system through experiments in the laboratory and in the operating room, itself.

\section{Description of the System}

\subsection{Magnetic and Optical Sensors}

Fastrak (Polhemus Inc., Colchester, VT), which utilizes of a transmitter and up to four receivers, was used as a magnetic sensor. The 3-D positions and 
orientations of the receivers can be measured with an accuracy of $0.8 \mathrm{~mm}$ RMS error if they are not affected by metallic objects. Fastrak's field of view is centered at the transmitter.

Optotrak (Northern Digital Inc., Waterloo, Ontario, Canada), which consists of a camera array and LED markers, was used as an optical sensor. The 3D positions of the LED markers can be measured with an accuracy of $0.1 \mathrm{~mm}$ RMS if the line-of-sight requirement is satisfied. Optotrak's field of view includes a $1.3 \times 1.3 \times 1.3 \mathrm{~m}^{3}$ volume whose center is at a distance of $2.25 \mathrm{~m}$ from the center of the camera array.

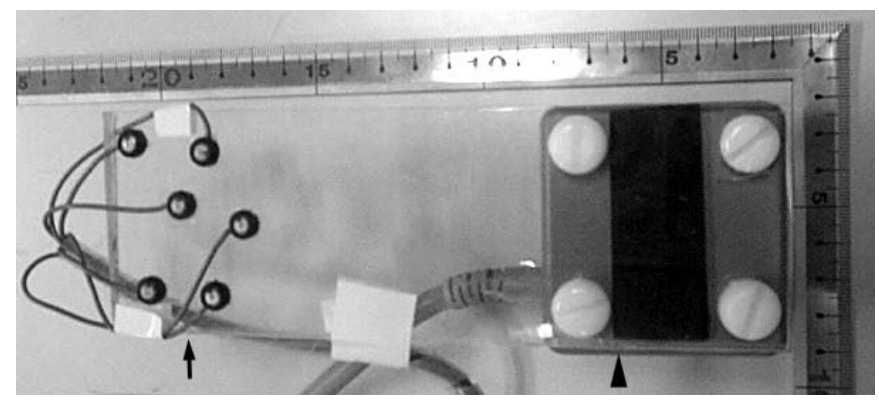

Fig. 1. Rigid body with LED markers attached to the Fastrak transmitter. Arrow: LED markers. Arrowhead: transmitter.

\subsection{Magneto-Optic Integration}

The Fastrak and Optotrak coordinate systems were integrated by moving Optotrak's LED markers on a rigid body attached to Fastrak transmitter (Fig. 1). Let $T_{o t}$ be the $4 \times 4$ matrix representing the transformation from the Optotrak coordinate system to the Fastrak coordinate system, and let $T_{t r}$ be the matrix representing the 3-D position and orientation of the receiver in the Fastrak coordinate system. By combining $T_{o t}$ and $T_{t r}$, the 3-D position and orientation of the receiver in the Optotrak coordinate system, $T_{o r}$, is estimated by

$$
T_{o r}=T_{o t} T_{t r} .
$$

Figure 2 shows the transformations of the coordinate systems in our hybrid sensor. In Eq. (1), $T_{t r}$ is directly measured and updated by Fastrak. $T_{o t}$ is obtained by

$$
T_{o t}=T_{o b} T_{b t},
$$

where $T_{o b}$ is the 3-D position and orientation of the rigid body attached to the transmitter, which is directly measured and updated by Optotrak. $T_{b t}$ is the 


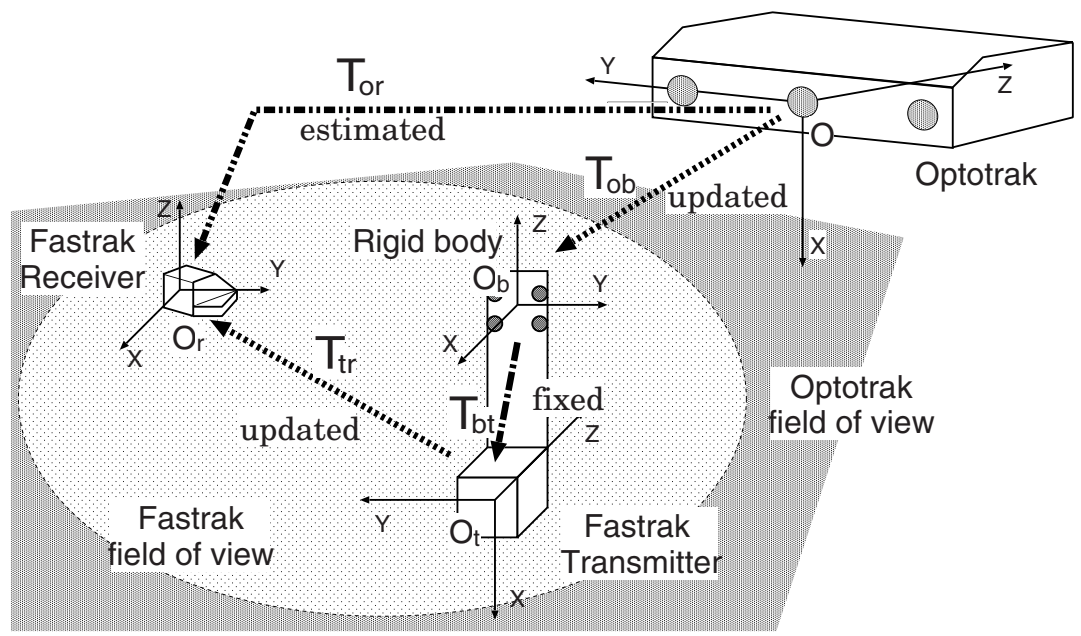

Fig. 2. Transformations between Fastrak and Optotrak coordinate systems.

fixed transformation from the rigid body to the Fastrak coordinate system, which needs to be estimated beforehand. The estimation of $T_{b t}$ is the only calibration needed for magneto-optic integration in our system.

\subsection{Calibration}

Let $\boldsymbol{x}_{m}$ and $\boldsymbol{x}_{o}$ be the $3-\mathrm{D}$ coordinates in the Fastrak and Optotrak coordinate systems, respectively. The relationship between them can be written as

$$
\boldsymbol{x}_{m}^{\prime}=\boldsymbol{x}_{o}^{\prime} T_{o b} T_{b t},
$$

where $\boldsymbol{x}_{m}^{\prime}$ and $\boldsymbol{x}_{o}^{\prime}$ are the homogeneous coordinates of $\boldsymbol{x}_{m}$ and $\boldsymbol{x}_{o}$, respectively. We acquire the Fastrak and Optotrak 3-D coordinates of control points uniformly distributed in the fields of view of both sensors. During the measurement of the control points, $T_{o b}$ is simultaneously measured by Optotrak. Given a sufficient number of control points, $T_{b t}$ is estimated using the least squares method. Data for the calibration needs to be acquired in an environment unaffected by metallic objects. Note that this calibration is performed only once, at the time when the rigid body is attached to the transmitter. Let $\boldsymbol{x}_{h}$ and $\boldsymbol{x}_{h}^{\prime}$ be the 3 -D coordinates measured by the hybrid sensor and its homogeneous coordinates, respectively. Using Eq. (3), $\boldsymbol{x}_{h}^{\prime}$ is given by

$$
\boldsymbol{x}_{h}^{\prime}=\boldsymbol{x}_{m}^{\prime} T_{b t}^{-1} T_{o b}^{-1}
$$

where $\boldsymbol{x}_{m}^{\prime}$ is the homogeneous coordinates of the direct measurement by Fastrak. $\boldsymbol{x}_{h}$ provides the 3 -D coordinates in the Optotrak coordinate system. 


\section{$3 \quad$ Experiments}

\subsection{Effects of Surgical Instruments}

We evaluated the effects of surgical instruments on the accuracy of the magnetic sensor through laboratory experiments using steel and aluminium rods with $100 \mathrm{~mm}$ in length and $9 \mathrm{~mm}$ in diameter and surgical instrument as sources of distortion. Steel and aluminium rods were respectively selected as typical ferromagnetic and non-magnetic metals. Figures 3(a) and 3(b) show typical setups for the experiment. The receiver was fixed and its position was assumed to be the origin; the transmitter and metallic object were placed at different positions. Let $D_{t r}$ be the position of the transmitter and $D_{m r}$ be that of the metallic object. The effect of the metallic object was evaluated by two settings. First, the transmitter was fixed at the position $D_{t r}=-30 \mathrm{~cm}$ and the position of the metallic object was varied as $D_{m r}=-20,-15,-10,-5,-3,3,5,10,15,20 \mathrm{~cm}$. Second, the metallic object was fixed at the position $D_{m r}=5 \mathrm{~cm}$ and the position of the transmitter was varied as $D_{t r}=-25,-20,-15,-10,-5,10,15,20,25,30 \mathrm{~cm}$. The bias was defined as $\left|\overline{\boldsymbol{x}}_{\text {metal }}-\overline{\boldsymbol{x}}_{\text {nometal }}\right|$, where $\boldsymbol{x}_{\text {metal }}$ and $\boldsymbol{x}_{\text {nometal }}$ are the 3 -D positions respectively measured with and without the metallic object in the same arrangement, and $\overline{\boldsymbol{x}}$ represents the average of different measurements for the same position.

Figures 3(c) and 3(d) show the results of the experiments. Thirty measurements were made at each position; the standard deviation was less than $0.1 \mathrm{~mm}$ at all the positions. In the first experiment, the bias was smaller when the metallic object was further from the receiver. When $D_{t r}=-30 \mathrm{~cm}$ and the metallic object was not positioned between the transmitter and the receiver, the bias was within $0.2 \mathrm{~mm}$ if the metal-receiver distance was more than $10 \mathrm{~cm}$, hereafter, the distance from object $\mathrm{A}$ to object $\mathrm{B}$ is referred to as the $\mathrm{A}-\mathrm{B}$ distance - for example, the distance from the receiver to the transmitter is termed the receiver-transmitter distance. In the second experiment, the bias was smaller when the transmitter was nearer to the receiver. When $D_{m r}=5 \mathrm{~cm}$ and the metallic object was not positioned between the transmitter and the receiver, the bias was within $0.4 \mathrm{~mm}$ if the transmitter-receiver distance was less than $15 \mathrm{~cm}$, and the bias was around $0.6 \mathrm{~mm}$ if the transmitter-receiver distance was $20 \mathrm{~cm}$. In both experiments, the bias was significantly smaller when the transmitter and metallic object were placed on opposite sides of the receiver, that is, when the metallic object was not positioned between the transmitter and the receiver.

\subsection{Effect of the OR Table}

Dual-Purpose Pen Probe for Accuracy Evaluation To evaluate the accuracy of the system, we compared the 3-D coordinates measured by the hybrid sensor with those measured by Optotrak. In order to simultaneously measure the 3-D position of the same point using the hybrid sensor and Optotrak, a dual-purpose pen probe was connected to both the Fastrak receiver and Optotrak's LEDs (Fig. 4(a)). Two aspects of the accuracy were evaluated - bias 


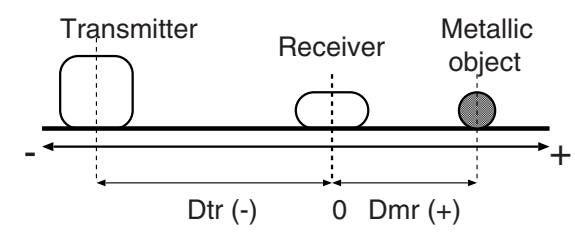

(a) Opposite side

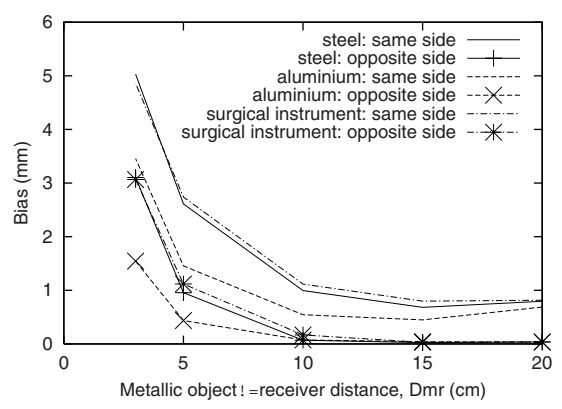

(c)

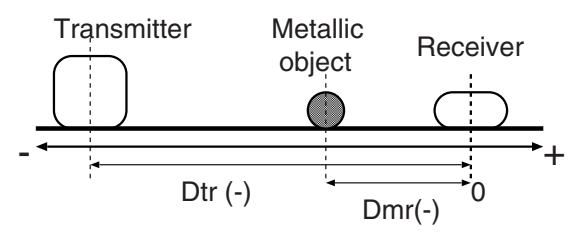

(b) Same side

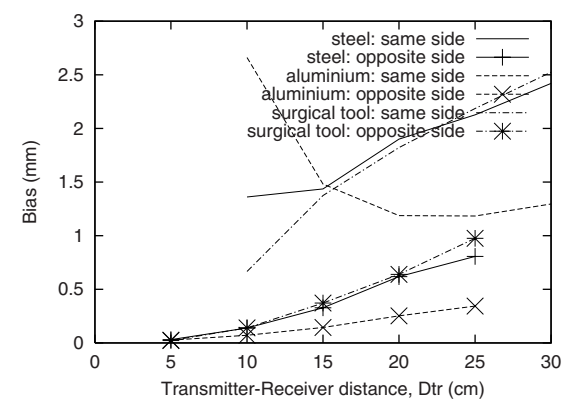

(d)

Fig. 3. Effects of surgical instruments. (a) Setup for experiments in which the transmitter is positioned on the opposite side of the receiver from the metallic object. The position of the receiver is regarded as the origin. $D_{m r}$ is the position of a metallic object. $D_{t r}$ is the position of the transmitter. (b) Setup for experiments in which the transmitter is positioned on the same side of the receiver as the metallic object. (c) Effects of $D_{m r}$ on bias, when $D_{t r}=-30 \mathrm{~cm}$. (d) Effects of $D_{t r}$ on bias, when $D_{m r}=5 \mathrm{~cm}$.

and precision. The bias was defined as $\left|\overline{\boldsymbol{x}}_{h}-\overline{\boldsymbol{x}}_{o}\right|$, where $\boldsymbol{x}_{h}$ and $\boldsymbol{x}_{o}$ are the 3 -D position measured by the hybrid sensor and directly by Optotrak, respectively. The precision was defined as the standard deviation in the measurements for the same point. Figures $4(\mathrm{~b})$ and $4(\mathrm{c})$ show the results of the pen-probe accuracy evaluation, which was carried out in an environment unaffected by metallic objects. The precision was maintained at about $0.1 \mathrm{~mm}$ in all the measurement ranges. The bias increased in proportion to the distance from the transmitter to the pen probe; when the distance was within $40 \mathrm{~cm}$, the bias was less than 1.0 $\mathrm{mm}$.

Laboratory Experiments The effect of a metal plate on the accuracy of the hybrid sensor was evaluated in laboratory experiments. Figure 5(a) shows 


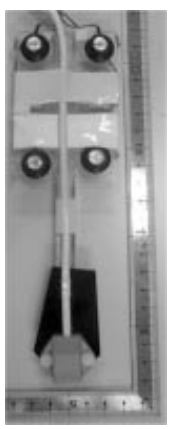

(a)

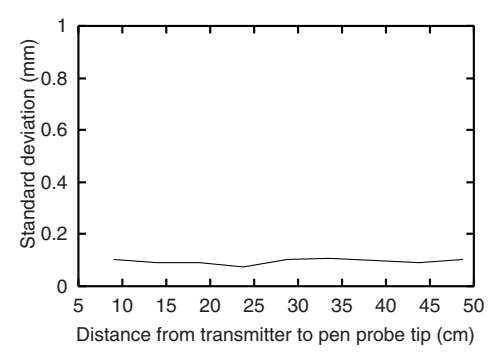

(b)

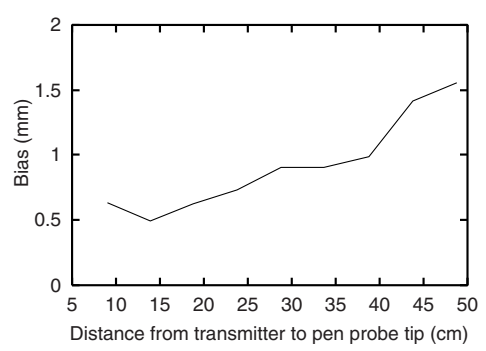

(c)

Fig. 4. Accuracy evaluation of dual-purpose pen probe. (a) Setup of Fastrak receiver and Optotrak LEDs. (b) Precision (standard deviation) evaluation. (c) Bias evaluation.

a typical experimental setup. A metal plate approximately $30 \times 80 \mathrm{~cm}^{2}$ was used. The transmitter and receiver were positioned above the plate at different metal plate-measurement plane distances, $D_{m p}$, and metal plate-transmitter distances, $D_{m t}$. Figure $5(\mathrm{~b})$ shows the effect of $D_{m t}$ on the bias when $D_{m p}$ was fixed at $19 \mathrm{~cm}$. The bias, $\left|\overline{\boldsymbol{x}}_{h}-\overline{\boldsymbol{x}}_{o}\right|$, was plotted for different values of $D_{t p}^{\prime}$. Figure $5(\mathrm{c})$ shows the effect of the metal plate-transmitter distance $D_{t p}^{\prime}$, when $D_{m t}$ was fixed at $40 \mathrm{~cm}$. When $D_{m t}=40 \mathrm{~cm}$ and $D_{m p}=37 \mathrm{~cm}$, the transmitterpen probe tip distance, $D_{t p}^{\prime}$, needed to be not more than around $20 \mathrm{~cm}$ to attain a bias within $1 \mathrm{~mm}$.

Operating Room Experiments The accuracy of the hybrid sensor was also evaluated in the OR environment. Figure 6(a) shows a typical experimental setup for the experiments. The transmitter was positioned at four different distances above the OR table plane. Figure 6(b) shows the effect of the transmitter-pen probe tip distance, $D_{t p}$, when $D_{m p}$ was fixed at $27 \mathrm{~cm}$. When the OR tabletransmitter distance was $40 \mathrm{~cm}$, the average bias of three points within $20 \mathrm{~cm}$ of $D_{t p}$ was $2.2 \mathrm{~mm}$; when the distance was $30 \mathrm{~cm}$, the average bias of six points within $15 \mathrm{~cm}$ was $2.0 \mathrm{~mm}$.

\section{Discussion and Conclusions}

We have proposed a new magneto-optic hybrid sensor configuration in which the 3-D position and orientation of the transmitter in a magnetic sensor system are tracked by an optical sensor, allowing the coordinate systems of the magnetic 
and optical sensors to be integrated and the transmitter to be positioned flexibly and adaptively. Experiments in the laboratory and in the operating room itself showed that inaccuracies arising from metallic objects could be reduced to acceptable levels by suitable placement of the transmitter and receivers.

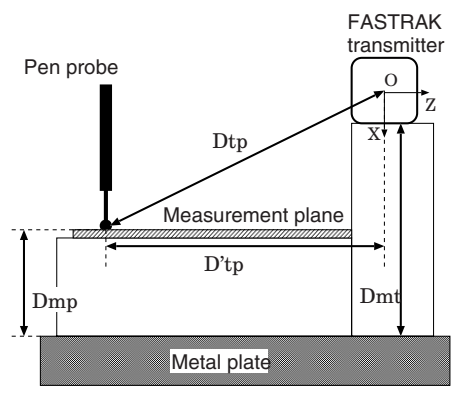

(a)

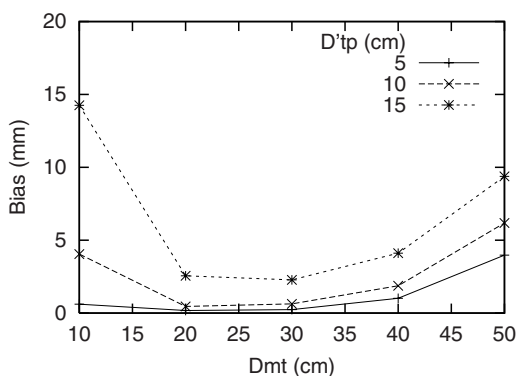

(b)

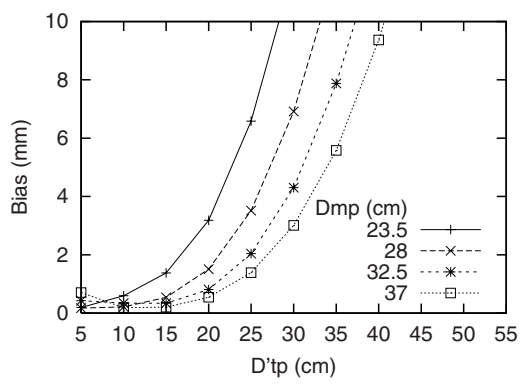

(c)

Fig. 5. Laboratory experiments for accuracy evaluation. (a) Setup for the experiments and definition of distances. $D_{m t}$ is the distance from the metal plate to the transmitter. $D_{m p}$ is the distance from the metal plate to the measurement plane. $D_{t p}$ is the distance from the transmitter to the pen probe tip. $D_{t p}^{\prime}$ is the length of the $D_{t p}$ projected onto the measurement plane. (b) Effects of $D_{m t}$ on bias when $D_{m p}=19 \mathrm{~cm}$. (c) Effects of $D_{t p}^{\prime}$ on bias when $D_{m t}=40 \mathrm{~cm}$.

Surgical instruments and the OR table are considered to be the two major sources of distortion. The experimental results on the effects of surgical instruments showed that the distortion was much smaller when the transmitter and the surgical instrument (or metallic object) were placed on opposite sides of the receiver. Thus, our hybrid system has the potential to minimize the distortion caused by surgical instruments since the placement of the transmitter can be 


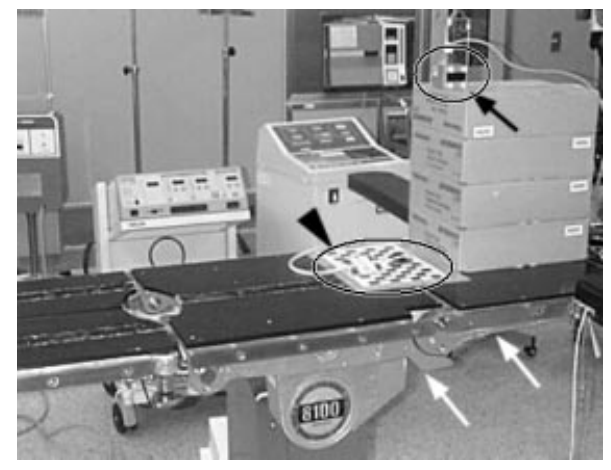

(a)

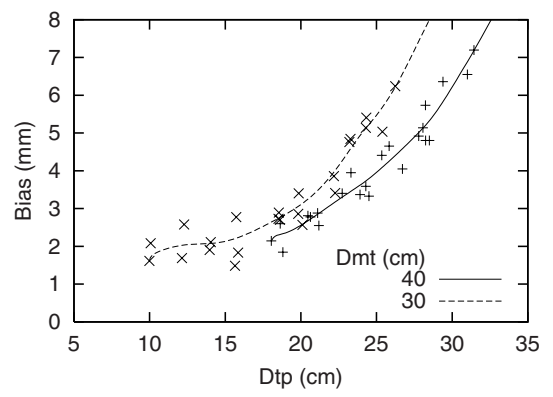

(b)

Fig. 6. Experiments in the operating room (OR). (a) Setup for experiments in the OR. Black arrow: transmitter. Black arrowhead: measurement plane. White arrow: metallic part of OR table. (b) Effects of $D_{t p}$ on bias when $D_{m p}=27 \mathrm{~cm}$.

changed intraoperatively. It should be noted that reducing the distortion caused by movable metallic objects such as surgical instruments is quite difficult when the pre-calibration method is used for distortion correction.

With respect to the effect of the OR table, it was found that the distance between the transmitter and the receiver needed to be relatively short to attain acceptable accuracy - for example, $20 \mathrm{~cm}$ to attain a bias of around $2 \mathrm{~mm}$ in the operating room experiment. Since acceptable accuracy is attainable within a limited area, say within a sphere with a radius of $20 \mathrm{~cm}$, flexibility in transmitter placement during clinical use is essential to track a sweet spot.

A potential criticism of the system relocating the transmitter may not be easy in a crowded operating environment. Because most commercial transmitters currently available are designed to be placed at a fixed position during a series of 3-D sensing procedures, they are relatively heavy and not suitable for flexible placement in the operating environment. However, transmitters can be made lighter and more compact. For instance, the transmitter used in the InstaTrak system (Visualization Technology Inc., Woburn, MA), which is used for surgical navigation during ENT surgery, is designed to be mobile, and is thus small and much lighter. This transmitter is attached to a head adaptor similar to a pair of spectacles, and moves with the patient's head motion to track the head-centered coordinate system. Flexible placement in the operating environment would be facilitated by using such a light and compact transmitter in the hybrid system.

Another problem concerns the design of the rigid body attached to the transmitter. To attain good accuracy, this needs to be relatively large, while the distance between the transmitter and the group of LED markers mounted on the 
rigid body should be small. However, a large rigid body is disadvantageous in terms of flexible transmitter placement and a short distance between the group of makers and the transmitter easily causes a blind spot. In our current design, the error due to the rigid body was relatively small compared with that due to distortion arising from the proximity to metallic objects. The tradeoffs involved in rigid body design will be further examined in future work.

Our ultimate goal is to use the hybrid sensor for 3-D ultrasound imaging, and we are now evaluating the effect of the ultrasound probe on the accuracy of the system. Preliminary experimental results indicate that the effect of the probe is less serious as that of the metallic objects examined in this paper. Future work will also include a quantitative comparison of 3-D ultrasound images obtained with the Optotrak system and the hybrid sensor.

Acknowledgment This work was partly supported by the Japan Society for the Promotion of Science (JSPS Research for the Future Program, JSPS Grant-inAid for Scientific Research (B)(2) 12558033, and Grant-in-Aid for JSPS Fellows).

\section{References}

[1] Y. Sato, M. Nakamoto, Y. Tamaki, et al. "Image guidance of breast cancer surgery using 3-D ultrasound images and augmented reality visualization". IEEE Trans. Med. Imaging, 17(5):681-693, 1998.

[2] T. Sasama, Y. Sato, N. Sugano, K. Nakahodo, S. Yoden, T. Nishii, K. Ohzono, T. Ochi, and S. Tamura. "Accuracy evaluation in computer assisted hip surgery". In Computer Assisted Radiology and Surgery: 13th Internaional Symposium and Exhibition (CARS'99), Paris, 1999.

[3] A. Kato, T. Yoshimine, T. Hayakawa, Y. Tomita, T. Ikeda, M. Mitomo, K. Harada, and H. Mogami. "A frameless, armless navigational system for computer-assisted neurosurgery". J Neurosurg, 5(74):845-849, 1991.

[4] A. State, G. Hirota, D. T. Chen, W. F. Garrett, and M. A. Livingston. "Superior augmented reality registration by integrating landmark tracking and magnetic tracking". In SIGGRAPH '96, pages 429-438, New Orleans, LA, 1996.

[5] W. Birkfellner, F. Watzinger, F. Wanschitz, G. Enislidis, M. Truppe, R. Ewers, and H. Bergmann. "Concepts and results in the development of a hybrid tracking system for CAS". In MICCAI '98, number 1496, pages 343-351, Cambridge, MA, 1998.

[6] W. Birkfellner, F. Watzinger, F. Wanschitz, R. Ewers, and H. Bergmann. "Calibration of tracking systems in a surgical environment". IEEE Trans Med Imaging, 17(5):737-742, 1998. 